\title{
ERAS in VATS-do we really need to follow the trend?
}

\author{
Hussein Elkhayat ${ }^{1}$, Diego Gonzalez-Rivas ${ }^{2,3}$ \\ ${ }^{1}$ Cardiothoracic Surgery, Assiut University, Faculty of Medicine, Assiut, Egypt; ${ }^{2}$ Department of Thoracic Surgery and Minimally Invasive Thoracic \\ Surgery Unit (UCTMI), Coruña University Hospital, Coruña, Spain; ${ }^{3}$ Department of Thoracic Surgery, Shanghai Pulmonary Hospital, Tongji \\ University School of Medicine, Shanghai 200433, China \\ Correspondence to: Hussein Elkhayat. Cardiothoracic Surgery Department, Assiut University Hospitals, Assiut, Egypt. Email: dr_khayat@hotmail.com. \\ Provenance: This is an invited article commissioned by the Editorial Office, Translational Lung Cancer Research.
}

Submitted Sep 15, 2019. Accepted for publication Oct 23, 2019.

doi: $10.21037 /$ tlcr.2019.11.02

View this article at: http://dx.doi.org/10.21037/tlcr.2019.11.02

Thoracic surgery specialty grows in a rapid consequence during the last two decades with a minimal invasive approach becoming more popular every day. The rationale was to improve the patient overall perioperative experience by improving his perception to pain and shorten the hospital stay this was defined as Improving patient outcomes by improving the quality of surgical care given (1). Trails to build up a fast track program Das-Neves-Pereira et al. publish their 5 years' experience with a rehabilitation program in which they use preoperative, intraoperative and postoperative measures to reduce pain and shorten hospital stay (2). The overall results were in favor of the fast track program, but their cohort did not contain any patient underwent minimal invasive approach despite they advices it in the inclusion criteria. They explain it as the technique is of VATS is not well established yet.

Surgeons start to notice that even in programs that contained VATS patients despite the minimal invasive techniques intraoperative, the overall hospital stay, and rate of pulmonary complication did not vary much as was expected comparing to the conventional thoracotomy. they realize that patient on the minimal invasive surgery get the same clinical pathway of the conventional surgery as nurses and health care personal accustomed on old protocols of patients leading to ambulate a patient on the fourth day of surgery and extensive analgesia were given to all patient. So, a clinical pathway to VATS patient start to develop as individual effort from high volume centers. The clinical pathway emphasis the importance of multidisciplinary team approach of patients including surgeons, nurses, anesthesiologists, physiotherapists. They divide the patient pathway into days with targets for each day and a responsible person for each step to be completed (3).

On the other side, colorectal surgery starts to develop the so called "enhanced recovery program after surgery; ERAS" which focus not only on the intraoperative phase and surgical technique but also its emphasis the importance of preoperative and postoperative care. Thoracic surgery shortly follow colorectal surgery and ERAS protocols starts to develop for thoracic surgery including items covering the preadmission, admission and counselling, preoperative adjustment, intraoperative techniques, anesthetics and postoperative pain management and care (4). The problem that thoracic surgeons report in recent publication on using of ERAS in thoracic surgery was that using ERAS protocols improve the overall patient perioperative experience and shortened hospital stay and decrease pulmonary complication on patient having conventional surgery but the results on VATS patients fail to demonstrate a statistically difference between ERAS and the standard care while still giving a better results compare to conventional group (5-7). This was explained by the fact that VATS itself is an enhanced recovery approach and patient can return much more easily from it to daily activity. Another point of view was that most of VATS program do the exact ERAS recommendation hence no major difference appear when ERAS program was implemented on those groups (5-7). An important notice here is that unlike ERAS programs for colorectal surgery, ERAS programs initially developed by institutions rather than societies representing its own practice and addressing their common pathway for thoracic surgery patients. This might render it difficult to be adopted on an international level and decreasing possibility of updating it on a regular basis. 
More recently in 2019 the ERAS society and ESTS develop guidelines for ERAS in thoracic surgery which contain an important item (8). The literature search (19662017) used Embase and PubMed to search medical subject headings including 'thoracic surgery', 'lung cancer surgery' and all perioperative ERAS items. They divide the program to preadmission, admission, preoperative, intraoperative and postoperative care. With each recommendation having level of evidence ranging from high to low and grade of recommendation ranging from strong to weak. Recommendations are based not only on the quality of evidence high, moderate, low and very low-but also on the balance between desirable and undesirable effects (8).

The main elements in the recent guidelines did not vary much than the previous institutional versions of ERAS $(5,6)$, the core target is to return a patient to his daily activity on the shortest time possible. This was planned for by the multidisciplinary team approach. The preoperative phase emphasis the importance of stop smoking 4 weeks before surgery and paying attention to patient over all nutritional status and weight loss as a strong recommendation with high level of evidence. Patient education, counselling and prehabilitation for patient with borderline lung function comes as a strong recommendation with weak evidence. Limit the fasting time to only 2 hours for clear fluids and 6 hours for solids before surgery and encourage the carbohydrate loading as it is found to improve wellbeing and reduce nausea and vomiting also no study investigate this results in thoracic surgery patients but these findings are considered valid for lung cancer patients given similarities in patient characteristics. Perioperative phase focus on antibiotic administration prior to skin incision, skin preparation using Chlorhexidine-alcohol rather than povidone-iodine solution, hair clipping and preventing hypothermia as hypothermia is associated with impaired drug metabolism, increased surgical site infection, cardiovascular morbidity and increased bleeding secondary to impaired hemostasis and its extended effect after surgery lead to postoperative shivering which increase oxygen consumption and worsen pain. Regional anesthesia is recommended to reduce postoperative opioid use. The use of paravertebral blockage and the combination of oral acetaminophen and NSAIDs should be used regularly in all patient unless contraindicated. On the intraoperative phase the evidence strongly recommends VATS approach in all patient with early stage lung cancer and to use muscle sparing thoracotomy for those will have a conventional surgery. Upon finishing the surgery, ERAS program recommends use of one chest tube if possible, use of digital drainage system, avoid routine use of negative suction and avoid routine use of urinary catheter.

ERAS program is a dynamic one despite this huge effort to organize and simplify it. future modifications with the implementation of it on everyday practice should be needed soon with more emerging evidence and audit. Modifications might include points like the fact that program is for thoracic surgery with special attention to lung resection which render the program large heterogenous groups of diseases with different requirements. The program mention VATS as a preferred approach but there was no difference between VATS and open thoracotomy in the pathway and care. The heterogenicity of diseases also reflected on approaches as guidelines did not recognize a difference between multiport, uniportal VATS and subxiphoid VATS. Future improvement which is likely to appear after audit on this new program and upon future publication with more strong evidence practice should address the fast track protocols of non-intubated VATS $(9,10)$, no chest tube (11), implementation of prehabilitation programs for all patient not just whose with borderline lung function and care for re admission after VATS.

\section{Acknowledgments}

None.

\section{Footnote}

Conflicts of Interest: The authors have no conflicts of interest to declare.

Ethical Statement: The authors are accountable for all aspects of the work in ensuring that questions related to the accuracy or integrity of any part of the work are appropriately investigated and resolved.

\section{References}

1. Osarogiagbon RU, D'Amico TA. Improving lung cancer outcomes by improving the quality of surgical care. Transl Lung Cancer Res 2015;4:424-31.

2. Das-Neves-Pereira JC, Bagan P, Coimbra-Israel AP, et al. Fast-track rehabilitation for lung cancer lobectomy: a fiveyear experience. Eur J Cardiothorac Surg 2009;36:383-91; discussion 391-2.

3. Sihoe ADL. Clinical pathway for video-assisted 
thoracic surgery: the Hong Kong story. J Thorac Dis 2016;8:S12-S22.

4. Schatz C. Enhanced Recovery in a Minimally Invasive Thoracic Surgery Program. AORN J 2015;102:482-92.

5. Martin LW, Sarosiek BM, Harrison MA, et al. Implementing a Thoracic Enhanced Recovery Program: Lessons Learned in the First Year. Ann Thorac Surg 2018;105:1597-604.

6. Brunelli A, Thomas C, Dinesh P, et al. Enhanced recovery pathway versus standard care in patients undergoing videoassisted thoracoscopic lobectomy. J Thorac Cardiovasc Surg 2017;154:2084-90.

7. Van Haren RM, Mehran RJ, Mena GE, et al. Enhanced Recovery Decreases Pulmonary and Cardiac Complications After Thoracotomy for Lung Cancer. Ann Thorac Surg 2018;106:272-9.

8. Batchelor TJP, Rasburn NJ, Abdelnour-Berchtold E, et

Cite this article as: Elkhayat $\mathrm{H}$, Gonzalez-Rivas D. ERAS in VATS-do we really need to follow the trend? Transl Lung Cancer Res 2019;8(Suppl 4):S451-S453. doi: 10.21037/ tlcr.2019.11.02 al. Guidelines for enhanced recovery after lung surgery: recommendations of the Enhanced Recovery After Surgery (ERAS(R)) Society and the European Society of Thoracic Surgeons (ESTS). Eur J Cardiothorac Surg 2019;55:91-115.

9. Holbek BL, Horsleben Petersen R, Kehlet H, et al. Fast-track video-assisted thoracoscopic surgery: future challenges. Scand Cardiovasc J 2016;50:78-82.

10. Gonzalez-Rivas D, Bonome C, Fieira E, et al. Nonintubated video-assisted thoracoscopic lung resections: the future of thoracic surgery? Eur J Cardiothorac Surg 2016;49:721-31.

11. El-Badry MM, Elkhayat H, Makhlouf GA, et al. Intraoperative removal of chest tube in video-assisted thoracoscopic procedures. Journal of the Egyptian Society of Cardio-Thoracic Surgery 2017;25:362-8. 